\title{
Study on the theory of three-dimensional limit analysis of soil and practical calculation
}

\author{
Bin $\mathrm{Li}^{\mathrm{a} 123456}$,Jianbao $\mathrm{Fu}{ }^{2345}$ \\ ${ }^{1}$ Guangxi Key Laboratory of Geomechanics and Geotechnical Engineering Guilin University of Technology, Guilin 541000, China \\ ${ }^{2}$ Tianjin Port Engineering Institute Co., Ltd. of CCCC First Harbor Engineering Co., Ltd., Tianjin 300222, China \\ ${ }^{3}$ Key Laboratory of Port Geotechnical Engineering, Ministry of Communications, PRC, Tianjin 300222, China \\ ${ }^{4}$.Key Laboratory of Tianjin Port Geotechnical Engineering, Tianjin 300222, China \\ ${ }^{5}$ CCCC First Harbor Engineering Company Ltd., Tianjin, 300461 China \\ ${ }^{6}$ China Key Laboratory of failure mechanism and safety control techniques of earth-rock dam of the Ministry of Water Resources, \\ Nanjing 210029;
}

\begin{abstract}
Based on the stress analysis of a point in space, the stress analysis of a point on a surface is performed, and then the relationship among the normal stress, the shear stress, and the stress component of a point on a surface and the first order partial derivative of the surface equation is deduced. Equilibrium equations of soil columns between the sliding surface and the top surface of the slope are established, which include differential equilibrium equation of force, equilibrium equation of force, and equilibrium equation of moment. These equilibrium equations and Coulomb yield condition can form the fundamental equations of three-dimensional slope stability analysis. Applying the supposition similar to that applied in the simplified Bishop method, a kind of three-dimensional slope analysis method can be obtained. An example is presented to show that the computation method is reasonable and applicable.
\end{abstract}

\section{INTRODUCTION}

The bearing capacity of the foundation, the stability of the slope and the earth pressure are the main problem in the engineering practice. For the problem of slope stability, no matter artificial slope or natural slope, the situation of landslide occurs is under three dimensional conditions, so the engineering meaning of 3D slope stability analysis is beyond doubt. Although the threedimensional stability analysis is of great significance, the related stability analysis methods and program development work are not meeting the actual needs. Most of the research work is limited to academic field. The existing 3D analysis methods have a lot of assumptions.

The problem of the bearing capacity of foundation is the shape of the foundation in engineering practice, the strip foundation (2D) is not exist, although in many cases can be simplified as a two-dimensional problem, or by two-dimensional approximate calculation; but it should be considered as $3 \mathrm{D}$ problem.

From the theoretical point of view, the problem of yield criterion is the basis of the theory of threedimensional limit analysis. However, up to now, there is no uniform definition for the stress field of the soil in the limit state. The fact is that no one type yield criterion can prove that the stress state is the most likely to cause damage of the soil.

There is no clear definition of the sliding surface. For example, the base yield criterion is a space stress

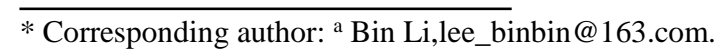

analysis, and no connection with the space surface; in fact, the limit state of stress field is connected with yield surface or sliding surface. The limit state of the soil is only along with the sliding surface, and the other surface is in a stable state (or limit state).

In addition, the existing yield criterion is only one of the equations that should be satisfied for the limit state of soil. It cannot form a complete set of basic equations together with the equilibrium equation. Under these conditions, only assumed conditions or other methods (constitutive models) can be used to determine the stress field.

It can be said that the research on the theory and application of three-dimensional soil limit analysis is behind engineering practice, and it isn't fully meeting the engineering needs.

\section{The establishment of the equation set}

\subsection{Research on the one point stress analysis in space}

(1) The basic relation of a point of stress in space

$$
\left.\begin{array}{c}
\sigma_{x} l+\tau_{x y} m+\tau_{x z} n=\tau l_{t}+\sigma l \\
\tau_{x y} l+\sigma_{y} m+\tau_{y z} n=\tau m_{t}+\sigma m \\
\tau_{x z} l+\tau_{y z} m+\sigma_{z} n=\tau n_{t}+\sigma n
\end{array}\right\}
$$




$$
\sigma_{x}, \sigma_{y}, \sigma_{z}, \tau_{x y}, \tau_{x z}, \tau_{y z} \text { are the stress }
$$
components. $\{l, m, n\}$ is a normal cosine of the inclined plane of a differential body, and $\left\{l_{t}, m_{t}, n_{t}\right\}$ is the direction cosine of the shear stress. $\sigma, \tau$ are the normal stress and shear stress on the oblique surface of the differential body.

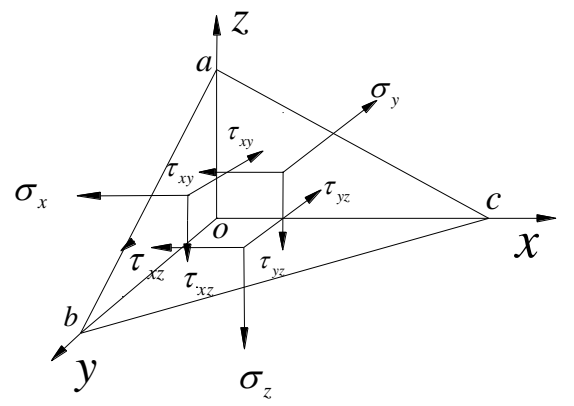

Figure 1. Diagram of the force on a differential body

This is the basic equation in the three-dimensional limit analysis theory. This basic equation is first put forward. It has important theoretical significance and wide application significance.

It is proved that the principal stress is the extreme value of the normal stress, and the principal shear stress is the extreme value of the shear stress.

The general form of the stress circle and the maximum stress circle are proposed for the first time.

$$
\begin{aligned}
& \tau^{2}+\left(\sigma-\sigma_{m}\right)^{2}=p_{t}^{2}, \\
& p_{t}^{2}=\left[\left(\sigma_{x}-\sigma_{m}\right) l+\tau_{x y} m+\tau_{x z} n\right]^{2} \\
& +\left[\tau_{x y} l+\left(\sigma_{y}-\sigma_{m}\right) m+\tau_{y z} n\right]^{2} \\
& +\left[\tau_{x z} l+\tau_{y z} m+\left(\sigma_{z}-\sigma_{m}\right) n\right]^{2}
\end{aligned}
$$

It is concluded that the maximum value of $p_{t}$ is the usual second stress invariants $\sqrt{J_{2}}$. On the stress surface of $\sigma \sim \tau, \tau^{2}+\left(\sigma-\sigma_{m}\right)^{2}=J_{2}$ is a circle of $\left(\sigma_{m}, 0\right.$ ), with a radius is $\sqrt{J_{2}}$. This is the maximum stress circle, which has obvious application significance: the shear stress of the stress circle is relatively large, the normal stress is relatively small, and the corresponding stress state is the most likely state of shear failure of the soil. It is demonstrated that the maximum stress circle is the real mole stress circle.

\section{2 yield criterion}

(1) Under the framework of the Coulomb yield function, a definition of the yield criterion is given.

If there is such a set of normal cosine: $f=0$, and all the other normal cosine, $f \leq 0$, the corresponding yield function of the group normal cosine is $\max \{f\}=0$, which is the yield criterion in the usual sense.

According to the limit state design principle, the stress state of the soil or foundation should be the ultimate stress state. In all possible stress states, the most likely state of failure is the ultimate stress state of the soil.

(2) Based on the real molar stress circle and the maximum value of Coulomb yield function, the extreme value yield criterion which accords to the limit state design principle is obtained.

$$
\sqrt{J_{2}}-\sigma_{m} \sin \varphi-c \cos \varphi=0
$$

The stress state corresponding to the maximum yield criterion is the most likely stress state of the soil which is most likely to occur shear failure, and the engineering significance is very clear.

(3) The expression of the stress component corresponding to the maximum yield criterion is obtained.

$$
\begin{aligned}
& \sigma_{x}+\frac{c}{\lambda}=\left(\sigma+\frac{c}{\lambda}\right)\left[1+2 \lambda l l_{t}+\left(1-l^{2}+l_{t}^{2}\right) \lambda^{2}\right] \\
& \sigma_{y}+\frac{c}{\lambda}=\left(\sigma+\frac{c}{\lambda}\right)\left[1+2 \lambda m m_{t}+\left(1-m^{2}+m_{t}^{2}\right) \lambda^{2}\right] \\
& \sigma_{z}+\frac{c}{\lambda}=\left(\sigma+\frac{c}{\lambda}\right)\left[1+2 \lambda n n_{t}+\left(1-n^{2}+n_{t}^{2}\right) \lambda^{2}\right] \\
& \tau_{x y}=\lambda\left(\sigma+\frac{c}{\lambda}\right)\left[\left(m l_{t}+l m_{t}\right)-\left(m l-m_{t} l_{t}\right) \lambda\right] \\
& \tau_{x z}=\lambda\left(\sigma+\frac{c}{\lambda}\right)\left[\left(n l_{t}+n_{t} l\right)-\left(n l-n_{t} l_{t}\right) \lambda\right] \\
& \tau_{y z}=\lambda\left(\sigma+\frac{c}{\lambda}\right)\left[\left(n m_{t}+n_{t} m\right)-\left(n m-n_{t} m_{t}\right) \lambda\right]
\end{aligned}
$$

The stress fields combine with equilibrium equations together to constitute a complete set of basic equations.

\subsection{The limit state of soil and the state criterion of ultimate stress field}

(1) For the first time, the stress analysis of the space is extended to a point stress analysis on the curved surface of the space, so that the stress analysis is related to the curved surface of the space, and such a curved surface of the space is the possible sliding surface. In order to study the relationship between the Coulomb yield function, the stress component and the sliding surface, it is convenient to further study the criterion of the ultimate stress field, including the sliding surface.

(2) The stress component in the limit state can be expressed as the normal stress, the yield surface and the function of $\alpha$.

$$
\begin{aligned}
& \sigma_{x}+\frac{c}{\lambda}=\left(\sigma+\frac{c}{\lambda}\right)\left[1+\lambda^{2}\left(\frac{1+h_{y}^{\prime 2}}{1+h_{x}^{\prime 2}+h_{y}^{\prime 2}}+\frac{\cos ^{2} \alpha}{1+A_{\alpha}^{2}}\right)\right. \\
& \left.-\frac{2 \lambda h_{x}^{\prime} \cos \alpha}{\sqrt{1+h_{x}^{\prime 2}+h_{y}^{\prime 2}} \sqrt{1+A_{\alpha}^{2}}}\right]
\end{aligned}
$$

(5a) 


$$
\begin{aligned}
& \sigma_{y}+\frac{c}{\lambda}=\left(\sigma+\frac{c}{\lambda}\right)\left[1+\lambda^{2}\left(\frac{1+h_{x}^{2}}{1+h_{x}^{\prime 2}+h_{y}^{\prime 2}}+\frac{\sin ^{2} \alpha}{1+A_{\alpha}^{2}}\right)\right. \\
& \left.-\frac{2 \lambda h_{y}^{\prime} \sin \alpha}{\sqrt{1+h_{x}^{\prime 2}+h_{y}^{\prime 2}} \sqrt{1+A_{\alpha}^{2}}}\right] \\
& (5 \mathrm{~b}) \\
& \sigma_{z}+\frac{c}{\lambda}=\left(\sigma+\frac{c}{\lambda}\right)\left[1+\lambda^{2}\left(\frac{h_{x}^{\prime 2}+h_{y}^{\prime 2}}{1+h_{x}^{\prime 2}+h_{y}^{\prime 2}}+\frac{A_{\alpha}^{2}}{1+A_{\alpha}^{2}}\right)\right. \\
& \left.+\frac{2 \lambda A_{\alpha}}{\sqrt{1+h_{x}^{\prime 2}+h_{y}^{\prime 2}} \sqrt{1+A_{\alpha}^{2}}}\right]
\end{aligned}
$$$$
\text { (5c) }
$$

$$
\begin{aligned}
& \tau_{x y}=-\lambda\left(\sigma+\frac{c}{\lambda}\right)\left[\lambda\left(\frac{h_{x}^{\prime} h_{y}^{\prime}}{1+h_{x}^{\prime 2}+h_{y}^{\prime 2}}-\frac{\cos \alpha \sin \alpha}{1+A_{\alpha}^{2}}\right)\right. \\
& \left.+\frac{h_{y}^{\prime} \cos \alpha+h_{x}^{\prime} \sin \alpha}{\sqrt{1+h_{x}^{\prime 2}+h_{y}^{\prime 2}} \sqrt{1+A_{\alpha}^{2}}}\right]
\end{aligned}
$$

$$
\begin{aligned}
& \tau_{x z}=\lambda\left(\sigma+\frac{c}{\lambda}\right)\left[\lambda\left(\frac{h_{x}^{\prime}}{1+h_{x}^{\prime 2}+h_{y}^{\prime 2}}+\frac{A_{\alpha} \cos \alpha}{1+A_{\alpha}^{2}}\right)\right. \\
& \left.+\frac{\cos \alpha-h_{x}^{\prime} A_{\alpha}}{\sqrt{1+h_{x}^{\prime 2}+h_{y}^{\prime 2}} \sqrt{1+A_{\alpha}^{2}}}\right]
\end{aligned}
$$

$(5 \mathrm{e})$

$$
\begin{aligned}
& \tau_{y z}=\lambda\left(\sigma+\frac{c}{\lambda}\right)\left[\lambda\left(\frac{h_{y}^{\prime}}{1+h_{x}^{\prime 2}+h_{y}^{\prime 2}}+\frac{A_{\alpha} \sin \alpha}{1+A_{\alpha}^{2}}\right)\right. \\
& \left.+\frac{\sin \alpha-h_{y}^{\prime} A_{\alpha}}{\sqrt{1+h_{x}^{\prime 2}+h_{y}^{\prime 2}} \sqrt{1+A_{\alpha}^{2}}}\right]
\end{aligned}
$$

(5f)

$$
A_{\alpha}=h_{x}^{\prime} \cos \alpha+h_{y}^{\prime} \sin \alpha
$$

$\alpha$ is the direction angle of the shear stress on the horizontal plane. Formula (5) is the limit stress field criterion.

The ultimate stress criterion and the equilibrium equations constitute a complete set of basic equations, lays theoretical basis for 3D limit analysis, limit analysis method of this theory fully complies with the limit state design principles, the complete set of equations is also first proposed.

\section{3 analysis of three dimensional slope}

The limit stress field criterion is introduced into the equilibrium equation, and the limit equilibrium equation is obtained. The equations have three unknown quantities: normal stress, yield surface and horizontal direction of failure. The derivation process is too complicated to explain in detail.

The equation along the failure surface is as follows:

$$
\begin{aligned}
& {\left[\frac{\partial}{\partial x}\left(\sigma+\frac{c}{\lambda}\right)+h_{x}^{\prime} \frac{\partial}{\partial z}\left(\sigma+\frac{c}{\lambda}\right)\right] C_{\alpha}+\left[\frac{\partial}{\partial y}\left(\sigma+\frac{c}{\lambda}\right)+h_{y}^{\prime} \frac{\partial}{\partial z}\left(\sigma+\frac{c}{\lambda}\right)\right] S_{\alpha}} \\
& -\lambda\left(\sigma+\frac{c}{\lambda}\right)\left(\frac{\partial h_{x}^{\prime}}{\partial x}+h_{x}^{\prime} \frac{\partial h_{x}^{\prime}}{\partial z}\right) \frac{1+h_{y}^{2}+C_{\alpha}^{2}}{1+h_{x}^{\prime 2}+h_{y}^{\prime 2}}+\lambda\left(\sigma+\frac{c}{\lambda}\right)\left(\frac{\partial h_{x}^{\prime}}{\partial y}+h_{y}^{\prime} \frac{\partial h_{x}^{\prime}}{\partial z}\right) \frac{h_{x}^{\prime} h_{y}^{\prime}-S_{\alpha} C_{\alpha}}{1+h_{x}^{\prime 2}+h_{y}^{2}} \\
& +\lambda\left(\sigma+\frac{c}{\lambda}\right)\left(\frac{\partial h_{y}^{\prime}}{\partial x}+h_{x}^{\prime} \frac{\partial h_{y}^{\prime}}{\partial z}\right) \frac{h_{x}^{\prime} h_{y}^{\prime}-S_{\alpha} C_{\alpha}}{1+h_{x}^{\prime 2}+h_{y}^{2}}-\lambda\left(\sigma+\frac{c}{\lambda}\right)\left(\frac{\partial h_{y}^{\prime}}{\partial y}+h_{y}^{\prime} \frac{\partial h_{y}^{\prime}}{\partial z}\right) \frac{1+h_{x}^{\prime 2}+S_{\alpha}^{2}}{1+h_{x}^{\prime 2}+h_{y}^{2}} \\
& =\gamma \frac{1}{1+\lambda^{2}}\left(h_{x}^{\prime} C_{\alpha}+h_{y}^{\prime} S_{\alpha}-\lambda\right)
\end{aligned}
$$

If the area enclosed by the surface $z=h(x, y)$ and the surface of the soil is closed area: $\Omega$, the virtual work equation is as follow:

$$
\begin{aligned}
& \iint_{D}\left(-p_{x} v_{x s}-p_{y} v_{y s}-p_{z} v_{z s}\right) \mathrm{d} x \mathrm{~d} y=\iiint_{\Omega} w_{z} \mathrm{~d} z \mathrm{~d} x \mathrm{~d} y \\
& -\iint_{D} \frac{c}{\lambda}\left[h_{x}^{\prime} v_{x}+h_{y}^{\prime} v_{y}-v_{z}\right]_{z=h} \mathrm{~d} x \mathrm{~d} y-\iiint_{\Omega}\left(\frac{\partial v_{x}}{\partial x}+\frac{\partial v_{y}}{\partial y}+\frac{\partial v_{z}}{\partial z}\right) \frac{c}{\lambda} \mathrm{d} z \mathrm{~d} x \mathrm{~d} y
\end{aligned}
$$

The imaginary work equation contains only the velocity component and the yield surface (or the sliding surface) without stress.

$$
\begin{aligned}
& C_{\alpha} \frac{\partial v}{\partial x}+S_{\alpha} \frac{\partial v}{\partial y}+\left(h_{x}^{\prime} C_{\alpha}+h_{y}^{\prime} S_{\alpha}\right) \frac{\partial v}{\partial z}+v\left[\frac{\partial C_{\alpha}}{\partial x}+\frac{\partial S_{\alpha}}{\partial y}+\frac{\partial}{\partial z}\left(h_{x}^{\prime} C_{\alpha}+h_{y}^{\prime} S_{\alpha}\right)\right] \\
& +v \lambda\left[\left(\frac{\partial h_{x}^{\prime}}{\partial x}+h_{x}^{\prime} \frac{\partial h_{x}^{\prime}}{\partial z}\right) \frac{1+h_{y}^{\prime 2}+C_{\alpha}^{2}}{1+h_{x}^{2}+h_{y}^{\prime 2}}-\left(\frac{\partial h_{y}^{\prime}}{\partial x}+h_{x}^{\prime} \frac{\partial h_{y}^{\prime}}{\partial z}\right) \frac{h_{x}^{\prime} h_{y}^{\prime}-S_{\alpha} C_{\alpha}}{1+h_{x}^{\prime 2}+h_{y}^{\prime 2}}\right. \\
& \left.-\left(\frac{\partial h_{x}^{\prime}}{\partial y}+h_{y}^{\prime} \frac{\partial h_{x}^{\prime}}{\partial z}\right) \frac{h_{x}^{\prime} h_{y}^{\prime}-S_{\alpha} C_{\alpha}}{1+h_{x}^{\prime 2}+h_{y}^{\prime 2}}+\left(\frac{\partial h_{y}^{\prime}}{\partial y}+h_{y}^{\prime} \frac{\partial h_{y}^{\prime}}{\partial z}\right) \frac{1+h_{x}^{\prime 2}+S_{\alpha}^{2}}{1+h_{x}^{2}+h_{y}^{2}}\right]=0
\end{aligned}
$$

(6c)

Formula (6c) is the velocity equation.

This is the first time to analyse the stability of the three dimensional slope under a hypothetical condition. The similar method has got according to the $2 \mathrm{D}$ slope simplified bishops method.

$$
\begin{aligned}
& M_{R}=\iint_{D} \frac{\left(w_{\gamma}+p_{z}-u\right) \tan \phi+c}{\frac{\Delta_{\alpha}}{\Delta_{h}}+\lambda_{F}\left(h_{x}^{\prime} \cos \alpha+h_{y}^{\prime} \sin \alpha\right)} \\
& \left\{\left[A_{1}\left(h-z_{R}\right)+A_{3}\left(y-y_{R}\right)\right]\left[\cos \alpha+h_{x}^{\prime}\left(h_{x}^{\prime} \cos \alpha+h_{y}^{\prime} \sin \alpha\right)\right]\right. \\
& +\left[A_{2}\left(h-z_{R}\right)-A_{3}\left(x-x_{R}\right)\right]\left[\sin \alpha+h_{y}^{\prime}\left(h_{x}^{\prime} \cos \alpha+h_{y}^{\prime} \sin \alpha\right]\right\} \mathrm{d} x \mathrm{~d} y \\
& \quad M_{0}=\iint_{D}\left(w_{\gamma}+p_{z}\right)\left\{h_{x}^{\prime}\left[A_{1}\left(h-z_{R}\right)+A_{3}\left(y-y_{R}\right)\right]\right. \\
& \left.\quad+h_{y}^{\prime}\left[A_{2}\left(h-z_{R}\right)-A_{3}\left(x-x_{R}\right)\right]\right\} \mathrm{d} x \mathrm{~d} y \\
& \quad+\iint_{D}\left\{p_{x}\left[A_{1}\left(s-z_{R}\right)+A_{3}\left(y-y_{R}\right)\right]\right. \\
& \left.\quad+p_{y}\left[A_{2}\left(s-z_{R}\right)-A_{3}\left(x-x_{R}\right)\right]\right\} \mathrm{d} x \mathrm{~d} y
\end{aligned}
$$

The safety factor is still calculated by $F_{s}=M_{R} / M_{0}$

a local load is on the $2 \mathrm{D}$ slope, which is a common case in engineering, It should be calculated in a threedimensional way. 


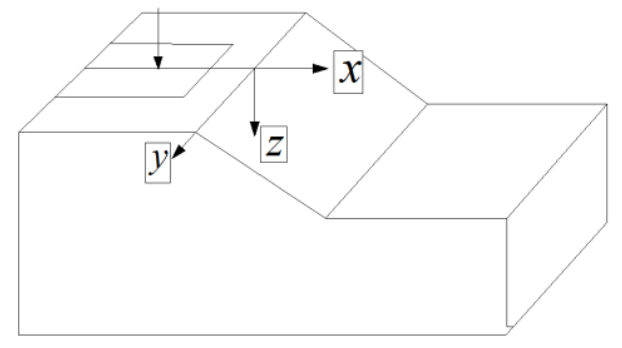

Figure 2. Diagram of local loading on Slope

Table 1. The safety factor of two-dimensional slope

\begin{tabular}{|c|c|c|c|c|c|c|c|}
\hline \multirow[t]{3}{*}{ sliding surface } & \multirow[t]{3}{*}{ condition } & \multirow{2}{*}{\multicolumn{2}{|c|}{$p_{z}=30 \mathrm{kPa}$}} & \multirow{3}{*}{ bishop } & \multicolumn{2}{|c|}{$p_{z}=60 \mathrm{kPa}$} & \multirow{3}{*}{ bishop } \\
\hline & & & Width of load & & \multicolumn{2}{|c|}{ width ofload } & \\
\hline & & $15 \mathrm{~m}$ & $30 \mathrm{~m}$ & & $15 \mathrm{~m}$ & $30 \mathrm{~m}$ & \\
\hline \multirow[t]{2}{*}{ Ellipsoidal surface } & $\varphi=20$ & 1.464 & 1.457 & 1.350 & 1.456 & 1.431 & 1.290 \\
\hline & $\varphi=25^{\circ}$ & 1.722 & 1.714 & 1.598 & 1.713 & 1.685 & 1.530 \\
\hline Ellipsoidal- & $\varphi=20^{\circ}$ & 1.460 & 1.444 & 1.350 & 1.452 & 1.428 & 1.290 \\
\hline $\begin{array}{l}\text { cylindrical- } \\
\text { ellipsoidal surface }\end{array}$ & $\varphi=25$ & 1.715 & 1.700 & 1.598 & 1.705 & 1.681 & 1.530 \\
\hline
\end{tabular}

Numerical examples show that the calculation results of this method are reasonable, This method is incomparable than other three dimensional slope stability analysis method who has a lot of assumptions.

\section{Conclusions}

(1) The velocity equation along with the sliding surface is proposed for the first time, which obtained stress equation, differential column force balance equation, torque balance equation, virtual work equation; these equations are universally applicable equation in threedimensional limit analysis. It provides a theoretical basis for the calculation of three-dimensional foundation bearing capacity and the slope stability.

(2) A variety of methods for solving threedimensional limit analysis are proposed. These methods are similar to two dimensional problem solving methods. That is to say, a scientific research method for threedimensional slope stability and foundation bearing capacity is found.

\section{Acknowledgement}

This research is financially supported by the project was funded by Guangxi Key Laboratory of Geomechanics and Geotechnical Engineering ( 16-KF-06) ;Open Foundation of Key Laboratory of Failure Mechanism and Safety Control Techniques of Earth-rock Dam of the Ministry of Water Resources (YK916002);

\section{References}

1. Hungr O, Salgado F M., Bryne P M. Evaluation Of A Three-Dimensional Method Of Slope Stability
Analysis[J]. Canadian Geotechnical Journal, 26:679686. (1989)

2. Feng Shu-ren, Feng Ding-xiang, Ge Xiu-run, Gu Xian-rong. 3D Limit Equilibrium Method For Slope Stability And Its Application[J]. Chinese Journal of Geotechnical Engineering, 21(6):657-661. (1999)

3. Zhang Junfeng. Extension Of Three-Dimensional Simplified Janbu's Method For Slope Stability Analysis[J]. Chinese Journal of Rock Mechanics and Engineering, 23 (17):2876-2881. (2004)

4. CHEN Zu-yu, MI Hong-liang, WANG Xiao-gang. A Three-Dimensional Limit Equilibrium Method For Slope Stability Analysis[J]. Chinese Journal of Geotechnical Engineering, 23(5):525-529. (2001)

5. ZHANG Jun-feng, WANG Si-ying, QI Tao. ThreeDimensional Spencer Method For Slope Stability Analysis[J]. Chinese Journal of Rock Mechanics and Engineering, 24(19): 3434-3439. (2005) 